\title{
BAFFLED CIRCULAR LOUDSPEAKER ARRAY WITH BROADBAND HIGH DIRECTIVITY
}

\author{
Mihailo Kolundžija, Christof Faller, and Martin Vetterli \\ School of Computer and Communication Sciences \\ Ecole Polytechnique Fédérale de Lausanne (EPFL), CH-1015 Lausanne, Switzerland \\ \{mihailo.kolundzija, christof.faller, martin.vetterli\}@epfl.ch
}

\begin{abstract}
Super-directional loudspeaker arrays can be used to achieve high directivity in a limited low-frequency range. As opposed to microphone arrays, the distance between the loudspeakers has to be relatively large, resulting in aliasing starting at relatively low frequencies. On the other hand, mounting a loudspeaker on a rigid baffle (e.g., a rigid cylinder or sphere) increases its directivity with frequency. Using super-directional array techniques at low frequencies and leveraging loudspeakers' increased directivity at high frequencies enables achieving high directivity both at low and high frequencies. The design of baffled circular loudspeaker arrays and an improved beamforming procedure for achieving high directivity in a broad frequency range is described.
\end{abstract}

Index Terms - loudspeakers, circular arrays, array signal processing, acoustic scattering, acoustic radiation effects

\section{INTRODUCTION}

Loudspeakers and loudspeaker systems with high directivity in a wide range of frequencies can be used in a variety of different applications, such as generation of spatial sound, mitigation of room effects, directional sound reproduction for public address systems, mobile terminals, etc. If such loudspeaker systems are to be of practical use, they should be compact, not overly complex, and feature wideband directivity.

A recently popular approach for building broadband loudspeakers with high directivity uses modulation of sound with an ultrasound carrier and exploits air propagation non-linearities for demodulation (e.g., see [1]). These systems have limited reproduction capabilities in terms of signal power and bandwidth. Beamforming techniques, which usually rely on far-field radiation characteristics of omnidirectional sources or receivers (e.g., see [2]), are inherently narrow-band and not suited for broadband applications, such as sound reproduction. Solutions proposed for broadband beamforming (e.g., see [3]), that are mostly concerned with transducer elements' placement and spacing, are also not appropriate, as they would require impractically sized arrays with a large number of transducers for achieving high broadband directivity. Even the super-directional techniques for designing microphone and loudspeaker arrays with frequencyindependent directional responses (e.g., see [4]) are of limited use in this case, as they would give appropriate performance only up to a relatively low aliasing frequency. This paper instead proposes the use of beamforming optimized considering a linear model of sound propagation and scattering.

This work was supported by the National Competence Center in Research on Mobile Information and Communication Systems (NCCR-MICS, http://wwww.mics.org), a center supported by the Swiss National Science Foundation under grant number 5005-67322.
During the last decade, a number of works that use the sound scattering properties of rigid bodies for designing microphone arrays have appeared, such as those of Meyer and Elko [5] and Teutsch and Kellermann [6]. These works leverage the effective increase in the microphone array aperture size, resulting from the scattering of rigid spheres and cylinders, for improving the signal-to-noise (SNR) performance at low frequencies and increasing the aliasing frequency.

In addition to improving the frequency response of the mounted transducers, rigid baffles modify their directional responses, making them more directive at higher frequencies. This property can be used for preserving directivity of a loudspeaker array in a wide range of frequencies. Namely, at low frequencies, the loudspeaker array can achieve a desired directivity with super-directional techniques, and at high frequencies, the increased directivity helps avoiding aliasing problems typical for arrays with transducers of relatively low directivity.

Another appealing property of the beamformer design approach presented in this paper is its practical applicability. Namely, it allows designing a broadband loudspeaker array with a well-behaved directional response by using measurements of loudspeaker responses at relatively few directions or points on a circle around the loudspeaker array.

The paper is organized as follows. Section 2 reviews the theoretical model of sound radiation from a loudspeaker mounted on a rigid cylinder. Section 3 shows a measurement-based approach to designing a beamforming loudspeaker array. Results of simulations are presented in Section 4, and conclusions are given in Section 5.

\section{SOUND FIELD OF A LOUDSPEAKER MOUNTED ON A CYLINDRICAL BAFFLE}

This section gives a theoretical result that serves as a basis for the broadband directional loudspeaker array design. Namely, it presents a theoretical model of a sound field emanating from a loudspeaker mounted on a rigid cylinder.

Note that the proposed beamforming approach is based on loudspeaker array measurement data and thus is not limited to the described model. However, the model is useful for determining array dimensions and getting indication of feasibility. The presented simulations use the model, since the process of building a loudspeaker array similar in dimensions to the one modeled in this paper is an ongoing work.

Consider the geometrical model of a loudspeaker mounted on a long rigid cylinder with radius $a$, shown in Fig. 1. The loudspeaker's membrane is modeled by a vibrating piston on the surface of a rigid cylinder, with length $2 L$ and circumferential width $2 a \alpha$. If the cylinder is of infinite length, then the sound field far away from the loud- 


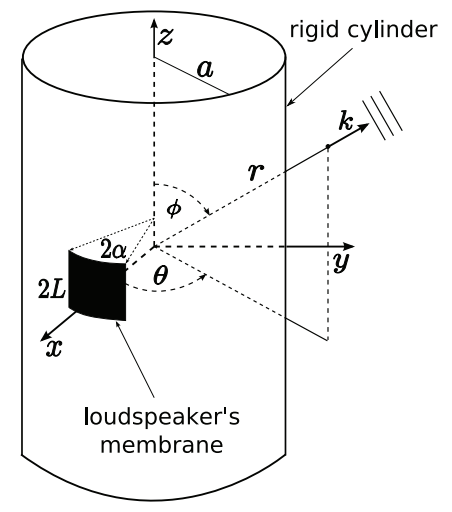

Fig. 1. Geometrical model of a loudspeaker mounted on a rigid cylinder. The loudspeaker's membrane is modeled as a vibrating piston of length $2 L$ and width $2 a \alpha$.

speaker is given by [7]

$$
p(\omega, r, \theta, \phi) \approx \frac{C \operatorname{sinc}(k L \cos \theta) e^{i k r}}{r \sin \theta} \sum_{n=-\infty}^{\infty} \frac{(-i)^{n} e^{i n \phi} \operatorname{sinc}(n \alpha)}{H_{n}^{\prime}(k a \sin \theta)}
$$

with

$$
C=\frac{4 b \alpha L \rho_{0} c}{2 \pi^{2}},
$$

where $r, \theta$, and $\phi$ are the standard spherical coordinates depicted in Fig. $1, \rho_{0}$ is the density of air, $c$ the speed of sound propagation, $b$ the piston's velocity, $k=\omega / c$ the wave number, and $H_{n}^{\prime}(x)$ the first derivative of the Hankel's function of the first kind.

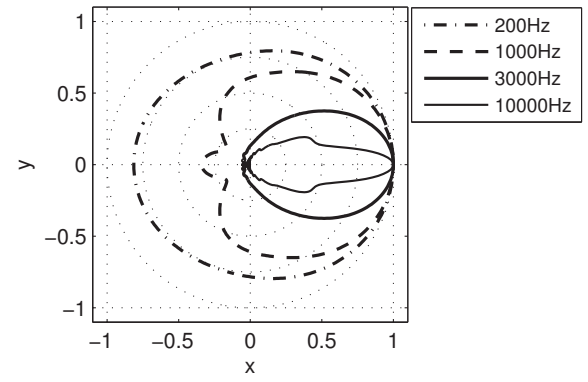

Fig. 2. Normalized directional responses at various frequencies of a piston vibrating on a surface of an infinitely long rigid cylinder.

Fig. 2 shows the directional responses of the described loudspeaker model at various frequencies with $a=0.1 \mathrm{~m}, L=2 \mathrm{~cm}$, and $\alpha=\pi / 20$. Note that the radiation characteristics do not change significantly if a finite-length cylinder is used, as was shown by Teutsch and Kellermann in [6].

The responses in Fig. 2 show that at low frequencies the directional response of a baffled loudspeaker is almost omnidirectional and that its directivity increases with frequency. This observation motivates the design of a broadband directional loudspeaker array. In particular, at low frequencies, multiple loudspeakers in a circular array can be combined with known super-directional beamforming techniques to achieve the desired response, and at higher frequencies, where super-directional techniques fail due to aliasing, a single loudspeaker pointing toward the desired direction can be used as a primary source, with the other loudspeakers only correcting the response in order to minimize the error relative to the desired directional response. This approach is described in the sequel.

\section{OPTIMIZED BEAMFORMER}

The focus of this part is on designing beamforming circular loudspeaker arrays. Note that the proposed approach is more general and can be applied to different configurations involving a baffle and multiple loudspeakers. ${ }^{1}$

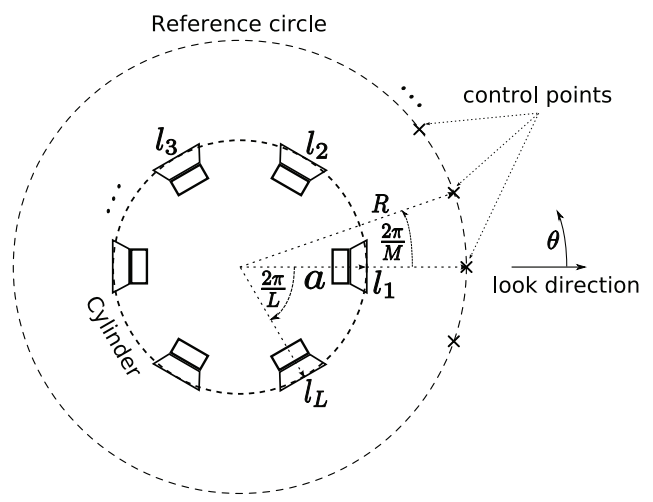

Fig. 3. Circular loudspeaker array configuration with reference circle on which the response is matched to a desired response.

Fig. 3 shows the considered circular array containing $L$ loudspeakers equally spaced on a circle with radius $a$ such that the $i$-th loudspeaker is located at angle $\theta_{i}=\frac{2 \pi}{L}(i-1)$. The effect of the previously described cylindrical baffle is implicitly included through directional response functions of the used loudspeakers. Furthermore, each loudspeaker points outwards, such that the response of the $i$-th loudspeaker is given by

$$
p_{i}(\omega, r, \theta, \phi)=p\left(\omega, r, \theta-\theta_{i}, \phi\right) .
$$

Since the focus is the loudspeaker array's response in the horizontal plane, the angle $\phi$ always equals $\pi / 2$, and is left out in the following analysis. Furthermore, it is assumed that the loudspeakers' directional responses $p(\omega, r, \theta)$ and the loudspeaker array's desired directional response $d(\theta)$ are band-limited periodic functions in $\theta$. Let $N$ denote the maximum order of $d(\theta)$ and $p(\omega, r, \theta)$.

\subsection{MMSE based beamformer design}

The assumption of the responses' band-limitedness, which is realistic for practical sound sources, enables designing a simple procedure for approximating the desired response $d(\theta)$ with the given loudspeaker array. Namely, by finding the minimum mean squared error (MMSE) fit to $d(\theta)$ of a linear combination of $p_{i}(\omega, r, \theta)$ on $M \geq 2 N$ equidistant points located on a circle with radius $R$ which is centered at the loudspeaker array's center, one achieves the MMSE fit on the entire circle. Additionally, one can put a constraint on loudspeaker filter gains in order to meet the physical limitations of the used loudspeakers, and prevent sound coloration in the look direction. The optimal beamforming filters at each frequency $\omega$ are

\footnotetext{
fle.

${ }^{1}$ One could similarly use loudspeaker arrays mounted on a spherical baf-
} 
then given by the solution of the following quadratic programming problem [8]:

$$
\begin{array}{cl}
\underset{\boldsymbol{h}}{\operatorname{minimize}} & \|\boldsymbol{d}-\boldsymbol{P}(\omega) \boldsymbol{h}\|_{2} \\
\text { subject to } & \left|h_{i}\right| \leq h_{\max }, i=1, \ldots, L \\
& \boldsymbol{p}_{1}(\omega) \boldsymbol{h}=d_{1},
\end{array}
$$

where $\boldsymbol{h}$ is a complex vector of length $L, \boldsymbol{d}$ is a complex vector ${ }^{2}$ of length $M$ whose elements are given by

$$
d_{i}=d(2 \pi(i-1) / M)
$$

$\boldsymbol{P}(\omega)$ is an $M \times L$ matrix whose elements are given by

$$
p_{i j}(\omega)=p(\omega, R, 2 \pi(i-1) / M-2 \pi(j-1) / L),
$$

and $\boldsymbol{p}_{1}(\omega)$ is the first row of the matrix $\boldsymbol{P}(\omega)$.

The loudspeaker array beamforming filters design can be summarized as follows:

1. Define a desired broadband directional response $d(\theta)$.

2. Assuming $d(\theta)$ and $p(\omega, r, \theta)$ are band-limited periodic functions of $\theta$ with maximum order $N$, measure the responses (propagation characteristics) from each loudspeaker to $M \geq$ $2 N$ equidistant points on a circle with radius $R$ (preferably $R \gg a$ ) centered at the loudspeaker array's center.

3. For each frequency $\omega$, find the beamformer filters' complex gains by solving (4). In practice, this amounts to computing the loudspeakers' complex gains at a number of frequencies determined by the filters' lengths, and obtaining the filters by computing the inverse discrete Fourier transform (e.g., see [9]).

\subsection{Beamformer design with phase alignment}

Although the design based on the criterion expressed in (4) gives the optimal fit to the desired response in the MSE sense respecting the physical constraints, it may be suboptimal in the energy sense, as it accounts for both magnitude and phase errors relative to the desired response. The main goal of a beamformer design for the loudspeaker array is steering the radiated energy, and phase errors are of only little importance. Therefore, a more appropriate beamformer design is the solution of the following optimization:

$$
\begin{array}{cl}
\underset{\boldsymbol{h}}{\operatorname{minimize}} & \|\boldsymbol{d} \cdot \overline{\boldsymbol{d}}-(\boldsymbol{P}(\omega) \boldsymbol{h}) \cdot(\overline{\boldsymbol{P}}(\omega) \overline{\boldsymbol{h}})\|_{2} \\
\text { subject to } & \left|h_{i}\right| \leq h_{\max }, i=1, \ldots, L \\
& \boldsymbol{p}_{1}(\omega) \boldsymbol{h}=d_{1},
\end{array}
$$

where $\boldsymbol{a} \cdot \boldsymbol{b}$ denotes an element-wise vector product (Hadamard product) of vectors $\boldsymbol{a}$ and $\boldsymbol{b}$, and $\overline{\boldsymbol{A}}$ denotes a vector or matrix with the complex conjugate elements of $\boldsymbol{A}$.

Unlike for the beamformer design via quadratic programming in (4), the solution of the beamformer design expressed in (6) can not be solved easily. Thus, in order to improve the desired response matching in the energy sense while using quadratic programming, we introduce an additional step of aligning phases of the desired response and the response of the loudspeaker that points toward the look direction of the beamformer:

$$
\boldsymbol{d}_{p a}(\omega)=|\boldsymbol{d}| \cdot \boldsymbol{e}(\omega),
$$

\footnotetext{
${ }^{2}$ The desired response is usually specified with real, non-negative gains.
}

where the phase alignment vector $\boldsymbol{e}(\omega)$ has elements

$$
e_{i}(\omega)=\frac{p(\omega, R, 2 \pi(i-1) / M)}{|p(\omega, R, 2 \pi(i-1) / M)|} .
$$

The beamformer is then obtained by solving (4) using at each frequency $\boldsymbol{d}_{p a}(\omega)$ as opposed to $\boldsymbol{d}$.

The motivation for the phase alignment is the following. At low frequencies, single loudspeaker responses do not have high directivity, and responses of different loudspeakers from Fig. 3 do not significantly differ in phase or magnitude on a reference circle. Thus, the beamformer obtained by solving (4) is close to optimal, ${ }^{3}$ even with slight changes in the desired response's phase. Toward high frequencies, single loudspeakers become more directive-and their responses differ more prominently in magnitude and phase- to the extent that a single loudspeaker can be used alone as the beamformer's output. Assuming a single loudspeaker is used starting from a certain frequency $f_{0}$, the beamforming filters obtained using phase-alignment - which biases the solution toward a more intensive use of the front loudspeaker-provides a smooth transition (fading) from beamforming to the use of a single loudspeaker. This smooth transition is desirable when designing broadband loudspeaker filters.

Fig. 4 shows a response matching improvement with phase alignment as frequency approaches $f_{0}$.
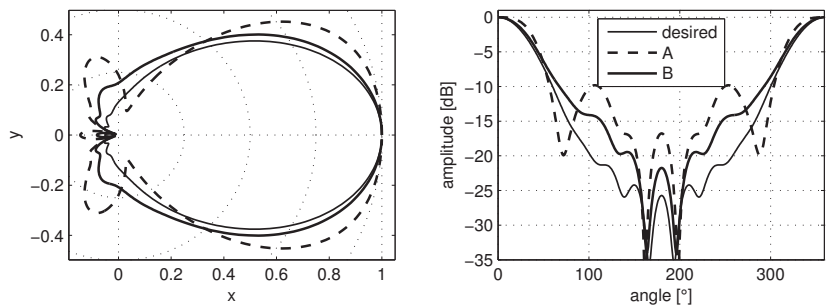

Fig. 4. Comparison of loudspeaker array's directional responses in linear (left) and logarithmic (right) scale obtained without (A) and with $(B)$ phase alignment for the frequency $2 \mathrm{kHz}$. The desired response is the directional response of a single loudspeaker mounted on a cylindrical baffle at frequency $f_{0}=3 \mathrm{kHz}$ (bold solid line in Fig. 2) and loudspeaker responses were computed using (1) with $a=10 \mathrm{~cm}, L=2 \mathrm{~cm}$, and $\alpha=\pi / 20$.

\section{SIMULATIONS}

This section presents the results from a number of simulations using a loudspeaker array with realistic dimensions. The simulated loudspeaker array consisted of six equally spaced loudspeakers mounted on the surface of a rigid cylinder. Loudspeaker responses were obtained using the model of a baffled loudspeaker expressed in (1). The piston, modeling the loudspeaker membrane, had the dimensions $L=2 \mathrm{~cm}$ and $\alpha=\pi / 20$, and the radius of the loudspeaker array was $a=10 \mathrm{~cm}$.

The control points for beamforming filter calculations were positioned equidistantly on a reference circle with radius $R=3 \mathrm{~m}$, concentric with the loudspeaker array. The number of reference points $M=13$ was chosen based on the assumption that the desired response and loudspeaker responses at different frequencies $\omega$ are band-limited periodic functions in $\theta$, with orders lower than or equal to six.

\footnotetext{
${ }^{3}$ The solution of (6) is considered as optimal.
} 
The used desired directional response was the directional response of a single loudspeaker mounted on a cylindrical baffle at frequency $f_{0}=3 \mathrm{kHz}$, as shown with a bold solid line in Fig. 2. The motivation behind this choice is that a single loudspeaker becomes directive enough such that it can be used alone as the beamformer's output above the frequency $f_{0}$.
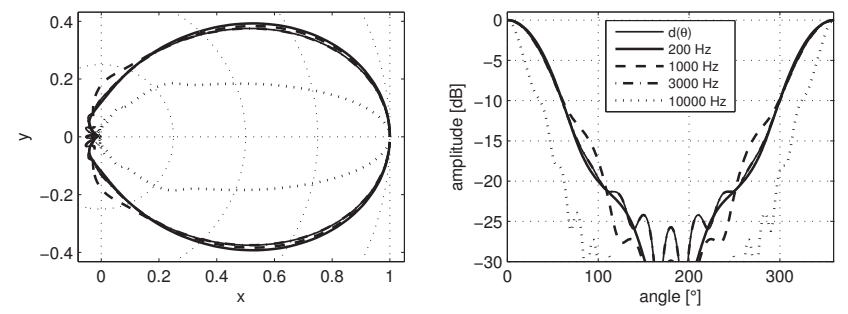

Fig. 5. Loudspeaker array directional responses in linear (left) and logarithmic (right) scale at various frequencies on the reference circle with radius $R=3 \mathrm{~m}$.

Fig. 5 shows directional responses-both in linear and logarithmic scale - of the loudspeaker array on the reference circle at various frequencies from $200 \mathrm{~Hz}$ to $10 \mathrm{kHz}$. As can be observed in Fig. 5, the directional responses of the loudspeaker array accurately match the desired response at frequencies up to about $3 \mathrm{kHz}$. At higher frequencies, the directional response of the loudspeaker array deviates from the desired response and becomes more directive due to the directivity increase of the one loudspeaker used at higher frequencies. This high-frequency directivity increase can be beneficial if high directivity is a design goal.

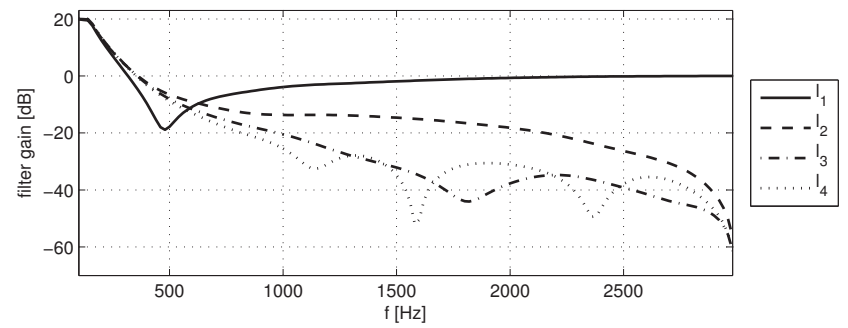

Fig. 6. Loudspeaker beamforming filters' frequency responses. The filters were normalized at all frequencies with a gain needed for the first loudspeaker alone to achieve the desired response in the beamformer's look direction $d(0)$.

Fig. 6 shows the beamforming filters of the first four loudspeakers. ${ }^{4}$ It can be observed that all loudspeakers have much larger gains at low frequencies (gains at frequencies below $200 \mathrm{~Hz}$ are by more than $20 \mathrm{~dB}$ larger than the gains at frequencies above $400 \mathrm{~Hz}$ ) than is needed for the first loudspeaker alone to achieve the desired response in the look direction, which is a known result from the super-directional array theory [4]. This could limit the working frequency range and the maximum low-frequency power of the designed loudspeaker array, depending on the physical limitations of the used loudspeakers. As the frequency increases, filter gains decrease due to the previously explained phase alignment, and above

\footnotetext{
${ }^{4}$ Due to symmetry relative to the beamformer's look direction, the fifth and the sixth loudspeaker have equal filters as the third and the second loudspeaker, respectively.
}

around $600 \mathrm{~Hz}$, the front loudspeaker becomes the main driver (it has the highest gain) while the gains of side and back loudspeakers decay toward zero at the frequency $f_{0}=3 \mathrm{kHz}$.

\section{CONCLUSIONS}

In this paper, it was shown how arrays of loudspeakers mounted on a rigid baffle can be used to achieve high directivity in a broad frequency range. Below the array's aliasing frequency, superdirectional processing yields relatively frequency-independent high directivity. At higher frequencies, the directivity caused by sound scattering due to the baffle is exploited to maintain high directivity and avoid aliasing. An MSE-optimized beamformer design procedure, based on measurements of the loudspeaker array relative to a few points on a reference circle, is proposed. Additionally, it was shown that the presented MSE-optimized beamformer design procedure can be improved by aligning phases between the desired response and the response of a loudspeaker pointing in the look direction of the beamformer.

The feasibility of the presented design was confirmed through simulations, which showed that an array of six loudspeakers mounted on a rigid cylinder with radius $a=10 \mathrm{~cm}$ can achieve high directivity in a wide frequency range, from $200 \mathrm{~Hz}$ to $10 \mathrm{kHz}$.

\section{REFERENCES}

[1] M. Yoneyama, J. Fujimoto, Y. Kawamo, and S. Sasabe, "The audio spotlight: An application of nonlinear interaction of sound waves to a new type of loudspeaker design," J. Acoust. Soc. Am, vol. 73, no. 5, pp. 1532-1536, 1983.

[2] B.D. Van Veen and K.M. Buckley, "Beamforming: A versatile approach to spatial filtering," IEEE ASSP Magazine, vol. 5, no. 2, pp. 4-24, 1988.

[3] D.B. Ward, R.A. Kennedy, and R.C. Williamson, "Theory and design of broadband sensor arrays with frequency invariant farfield beam patterns," J. Acoust. Soc. Am, vol. 97, no. 2, pp. 1023-1034, 1995.

[4] Gary W. Elko, "Superdirectional microphone arrays," in Acoustic signal processing for telecommunication, pp. 181-238. Kluwer Academic Publishers, 2000.

[5] J. Meyer and G. Elko, "A highly scalable spherical microphone array based on an orthonormal decomposition of the soundfield," in Proceedings (ICASSP'02), 2002, vol. 2.

[6] H. Teutsch and W. Kellermann, "Acoustic source detection and localization based on wavefield decomposition using circular microphone arrays," J. Acoust. Soc. Am, vol. 120, no. 5, pp. 2724-2736, 2006.

[7] E.G. Williams, Fourier acoustics: sound radiation and nearfield acoustical holography, Academic Press, 1999.

[8] S.P. Boyd and L. Vandenberghe, Convex optimization, Cambridge university press, 2004.

[9] M. Kolundžija, C. Faller, and M. Vetterli, "Designing Practical Filters For Sound Field Reconstruction," in Preprint 127th Conv. Aud. Eng. Soc., Oct. 2009. 